\title{
NORMALITY AND TERMINALITY IN THE ELEMENTARY SUBGROUPS OF STEINBERG GROUPS OVER RINGS
}

\author{
JAMES F. HURLEY
}

\begin{abstract}
The elementary subgroups of Steinberg groups over commutative rings with identity are constructed internally in the manner used by Chevalley and Steinberg over fields. A uniform method is given for the construction of normal subgroups of these elementary groups. Terminality results obtained by Spitznagel over fields are shown to follow in the same way over rings in which 2 can be inverted.
\end{abstract}

1. Introduction. In [8] we have shown how the fundamental construction given by Chevalley [3] can be carried out equally as well over commutative rings $R$ with identity as over fields. The resulting groups $G_{R}$ turn out to be important (see for example [2, pp. 94-127]) elementary subgroups of the classical special linear, symplectic and orthogonal groups modulo their centers. In the context of the Chevalley-Demazure theory ([4], [5]) these groups are usually referred to as the elementary subgroups of the Chevalley groups defined over $R$ (see for example [1] and [12]). In many cases these elementary subgroups coincide with the full Chevalley groups over $R[9$, p. 102], the most familiar example being of course when $R$ is a field.

We have further given a uniform method for the construction of large classes of normal subgroups of the groups $G_{R}$, a method which consists basically of exponentiating ideals of the corresponding Chevalley algebras [7] over $R$. Since the ideal structure of these Chevalley algebras has been completely determined in terms of the ideal structure of the rings and the root systems of the underlying simple Lie algebras over the complex numbers, we thus have been able to directly relate the normal structure of elementary subgroups of Chevalley groups over rings to classical Lie and commutative algebra.

In the present paper we show that the same uniform procedure can be applied to the elementary subgroups of the Steinberg groups [13] to again

Received by the editors August 10, 1971 and, in revised form, October 19, 1971.

AMS 1970 subject classifications. Primary 20G35, 17B20, 20H25; Secondary 20D15, $20 \mathrm{~F} 40$.

Key words and phrases. Chevalley group, Steinberg group, terminal nilpotent group, Chevalley algebra, elementary subgroups, classical groups over rings, root systems, Lie algebras. 
construct normal subgroups corresponding to ideals in the Chevalley algebras over $R$.

In closing we note that the terminality results obtained by Spitznagel [11] for Steinberg groups over fields carry over to the elementary subgroups of Steinberg groups over rings in which the key structure constant 2 is invertible.

2. Construction of Chevalley algebras and groups, Steinberg groups, and elementary subgroups. Full details of the construction of Chevalley algebras and groups and elementary subgroups over rings may be found in [8] and [14]. One starts with a finite dimensional simple Lie algebra $L$ over the complex field, a Cartan subalgebra $H$ of dimension $n$, a set $S$ of nonzero roots relative to $H$ ordered in a manner consistent with heights, the set $P$ of positive roots relative to this ordering, and a Chevalley basis $B=\left\{e_{r} \mid r \in S\right\} \cup\left\{h_{1}, \cdots, h_{n}\right\}$.

In [3] and the present paper, we may define $L_{\mathbf{Z}}$ to be the free abelian group on $B$, and $L_{R}=R \otimes_{\mathbf{z}} L_{\mathbf{z}}$ is called the (adjoint) Chevalley algebra of $L$ over $R$. Now $x_{r}(t)=\exp \left(\operatorname{ad} t e_{r}\right)=\sum_{0}^{\infty} t^{n}\left(\operatorname{ad} e_{r}\right)^{n} / n$ ! acts on $L_{R}$ meaningfully if $t \in R$. The one parameter group $X_{r}=\left\{x_{r}(t) \mid t \in R\right\}$ is well defined, and the group $G_{R}$ generated by all the one parameter groups $X_{r}$ is called the elementary subgroup of the Chevalley group of $L$ over $R$.

In $G_{R}$ the group $U_{R}$ generated by all $x_{r}(t)$ with $r$ positive is nilpotent. A central series consists of the groups $U_{R(k)}$ generated by those $x_{r}(t)$ such that $r$ has height at least $k[14$, p. 26].

Suppose now that the Lie algebra $L$ is such that its Dynkin diagram has a symmetry $r \rightarrow \bar{r}$ of order two or three, and suppose the ring $R$ has an automorphism $t \rightarrow \bar{t}$ of the same order. Then $\sigma: G_{R} \rightarrow G_{R}$ given by $\sigma\left(x_{r}(t)\right)=$ $x_{r}^{-}(\bar{t})$ is an automorphism. Let $V_{R}$ be the group generated by all $x_{r}(t)$ with $r$ a negative root. Let $U_{R}^{1}=\left\{u \in U_{R} \mid \sigma(u)=u\right\}$ and $V_{R}^{1}=\left\{v \in V_{R} \mid \sigma(v)=v\right\}$. Then $G_{R}^{1}$, the subgroup of $G_{R}$ generated by $U_{R}^{1}$ and $V_{R}^{1}$, is called the elementary subgroup of the Steinberg group of $L$ over $R$. We remark that $G_{R}^{1}$ is not in general the subgroup of those elements of $G_{R}$ fixed by $\sigma[13, \mathrm{p}$. 891]. We also remark that if $U_{R(k)}^{1}=U_{R} \cap U_{R(k)}$, then we get a central series in $U_{R}^{1}$ [11, p. 400].

A basic tool in studying Steinberg groups over fields is the collection $P^{1}$ of sets of roots of types (1), (2), and (3), and the reader is referred to $[13$, p. 876] for definitions and details.

3. Normal subgroups of $G_{R}$. We now state the main result of [8]. Let $I$ be an ideal of $L_{R}$ not wholly contained in $H_{R}=R \otimes_{\mathrm{z}} H_{\mathrm{Z}}$ (where $H_{\mathrm{z}}$ is the additive abelian group generated by $\left.h_{1}, h_{2}, \cdots, h_{n}\right)$. Let $G_{I}$ denote the subgroup of $G_{R}$ generated by those $x_{r}(t)$ for which $t e_{r} \in I(t \in R, r \in S)$ and all iterated conjugates $C_{k}\left(C_{k-1}\left(\cdots\left(C_{1}\left(x_{r}(t)\right)\right) \cdots\right)\right)$. Here $C_{1}$ denotes 
conjugation by $x_{-r}\left(u_{1}\right)$ for some $u_{1} \in R, C_{2}$ denotes conjugation by $x_{r}\left(u_{2}\right)$ for some $u_{2} \in R, C_{3}$ denotes conjugation by $x_{-r}\left(u_{3}\right)$ for some $u_{3} \in R$, etc. Then $G_{I}$ is a normal subgroup of $G_{R}$.

The proof of this result is based on a careful study of the basic commutator lemma in $G_{R}$, which in the present context takes the following form [14, p. 24].

LEMMA. If $r$ and $s$ are linearly independent roots, then $\left(x_{r}(t), x_{s}(u)\right)=1$ in case $r+s$ is not a root, and $\left(x_{r}(t), x_{s}(u)\right)=x_{r+s}( \pm t u)$ in case $r+s$ is a root.

4. Normal subgroups of $G_{R}^{1}$. Let $I$ be an ideal of $L_{R}$ not wholly contained in $H_{R}$. Then we have the following result.

THEOREM 1. Let $G_{I}^{1}$ be the subgroup of $G_{R}^{1}$ generated by all $x_{r}(t)$ in $G_{R}^{1}$ such that $t e_{r} \in I$ and by all iterated conjugates defined above. Then $G_{I}^{1}$ is normal in $G_{R}^{1}$.

Proof. If we knew that $G_{I}^{1}=G_{I} \cap G_{R}^{1}$, then the result would follow immediately from the fact that $G_{I}$ is normal in $G_{R}$. But since $G_{R}^{1}$ is not in general the subgroup of fixed points under $\sigma$ in $G_{R}$, we cannot assert this. $G_{R}^{1}$ is, however, generated by elements $x_{r}(t)$ in $U_{R}^{1}$ or $V_{R}^{1}$, so it is sufficient to check that the conjugates of the generators of $G_{I}^{1}$ by generators of $G_{R}^{1}$ fall back in $G_{I}^{1}$. Suppose then that $x_{s}(u) \in G_{I}^{1}$ and $x_{r}(t) \in G_{R}^{1}$ with $r \neq \pm s$. Then we have $x_{r}(t) x_{s}(u) x_{r}(-t)=x_{r+s}( \pm t u) x_{s}(u)$ or $x_{r}(t) x_{s}(u) x_{r}(-t)=x_{s}(u)$ according as $r+s$ is or is not a root. In the latter case, the conjugate is still in $G_{I}^{1}$. In the former case, since $r$ and $s$ are invariant under the symmetry of the root system of $L$ corresponding to $\sigma$, so is $r+s$. Thus the right-hand side is in $G_{I}^{1}$ in view of the fact that $e_{r+s} \in I$ whenever $e_{s}$ belongs to $I$. If $r=s$, then $x_{r}(t) x_{r}(u) x_{r}(-t)=x_{r}(u) \in G_{I}^{1}$. If $r=-s$, then $x_{r}(t) x_{-r}(u) x_{r}(-t)$ is one of the generators of $G_{I}^{1}$. Thus the conjugates of simple root element generators of $G_{I}^{1}$ by generators of $G_{R}^{1}$ are in $G_{I}^{1}$. Finally, in case one of the iterated conjugate generators of $G_{I}^{1}$ is conjugated by an $x_{s}$ with $s \neq \pm r$, the result falls back in $G_{I}^{1}$ by repeated application of the commutator lemma as in the proof of the normality of $G_{I}$ in $G_{R}$ [8].

A partial converse of Theorem 1 holds in the present setting.

THEOREM 2. If $N$ is a normal subgroup of $G_{\mathbf{Z}}^{1}$ and $I \subseteq L_{\mathbf{Z}}$ is the ideal generated by $D=\left\{d_{r} e_{r} \mid r \in S\right\}$, where $d_{r}=$ g.c.d. $\left\{n \in \mathbf{Z} \mid x_{r}(n) \in G_{R}^{1}\right.$ is a factor of some element in $N\}$, then $N \subseteq G_{I}^{1}$.

Proof. All $x_{r}\left(d_{r}\right) \in G_{I}^{1}$ since they are among the generators. If $y \in N$, then $y$ is a product of factors $x_{r}(n)$, where $d_{r}$ divides $n$. Thus $n=d_{r} q$ for some integer $q$. So $x_{r}(n)=x_{r}\left(d_{r}\right)^{q}$. Thus $y \in G_{I}^{1}$.

5. Terminality in $G_{R}^{1}$. Evens [6] introduced the notion of a terminal nilpotent group. If $G$ is nilpotent of class $m$, then it is terminal if 
$N / \Gamma_{m+1}(N) \cong G$ for any nilpotent group $N$ implies $\Gamma_{m+1}(N)=\{0\}$. Evens showed that nonabelian $p$-Sylow subgroups of the finite classical groups are terminal provided the underlying field has characteristic other than $p$. Spitznagel was able to remove this restriction by showing that the maximal unipotent subgroups of Chevalley and Steinberg groups are terminal if the field is not of characteristic 2 and the Lie algebra is of rank at least 4 ([10], [11]). We note here that the results of [11] extend to the elementary subgroups of Steinberg groups over rings in which 2 can be inverted.

THEOREM 3. Let $U_{R}^{1}$ be as defined in $\S 2, L$ of type $A_{n}$ ( $n$ odd), $D_{n}$, or $E_{6}$, and 2 anit in $R$. Then

(1) $U_{R}^{1}$ is generated by the groups $U_{i}$ for $\left\{S_{i}\right\}$ a fundamental system of sets of positive roots of the respective types $C_{k}\left(k=\frac{1}{2}(n+1)\right), B_{n-1}$, or $F_{4}$ identified with $P^{1}$.

(2) The groups $U_{R(k)}^{1}$ form the lower central series of $U_{R}^{1}$. Thus the class of $U_{R}^{1}$ is the class of $U_{R}$, namely the height of the highest root in the root system of type $C_{k}\left(k=\frac{1}{2}(n+1)\right), B_{n-1}$, or $F_{4}$ identified with $P^{1}$.

Proof. If $R$ is a field, then this is just Theorem 1 of [11]. An examination of the proof in [11] reveals that only properties of root systems and the invertibility of 2 were needed. Since we have added the hypothesis that 2 be invertible in $R$, the result will follow from the argument given in [11].

THEOREM 4. In Theorem 3 suppose that if $L$ is of type $A_{n}$, then $n \geqq 7$ is odd and if $L$ is of type $D_{n}$, then $n \geqq 5$. Then $U_{R}^{1}$ is terminal nilpotent.

Proof. The proof of this result for $R$ a field [11, Theorem 2] required only the invertibility of 2 , the multiplicative properties of a Chevalley basis, and the finding of roots with certain technical properties in each of the types $C_{k}, k=\frac{1}{2}(n+1), B_{n-1}$, and $F_{4}$. Thus the result will follow in the present context from the argument given in [11].

The case of $A_{n}, n$ even, is more complicated, as usual in Steinberg groups. However, Spitznagel is able to establish the results of Theorems 3 and 4 in this case as well without using any field properties beyond the invertibility of 2 . This result as well then carries over to the present setting.

\section{REFERENCES}

1. E. Abe, Chevalley groups over local rings, Tôhoku Math. J. (2) 21 (1969), 474-494. MR 41 \#3483.

2. H. Bass, J. Milnor and J.-P. Serre, Solution of the congruence subgroup problem for $\mathrm{SL}_{n}(n \geqq 3)$ and $\mathrm{Sp}_{2 n}(n \geqq 2)$, Inst. Hautes Études Sci. Publ. Math. No. 33 (1967), 59-137. MR 39 \#5574.

3. C. Chevalley, Sur certains groupes simples, Tôhoku Math. J. (2) 7 (1955), 14-66. MR 17, 457. 
4. C. Chevalley, Certains schémas de groupes semisimples, Séminaire Bourbaki 1960/61, Secrétariat mathématique, Paris, 1961. MR 27 \#1339.

5. M. Demazure and A. Grothendieck, Séminaire de géométrie algébrique: Schémas en groupes, Inst. Hautes Études Sci. 1963/64, Lecture Notes in Math. nos. 151, 152, 153, Springer-Verlag, Berlin and New York, 1970/71.

6. L. Evens, Terminal p-groups, Illinois J. Math. 12 (1968), 682-691. MR 39 \#2875.

7. J. Hurley, Ideals in Chevalley algebras, Trans. Amer. Math. Soc. 137 (1969), 245258. MR 38 \#5870.

8. - Some normal subgroups of elementary subgroups of Chevalley groups over rings, Amer. J. Math. 93 (1971), 1059-1069.

9. H. Matsumoto, Subgroups of finite index in certain arithmetic groups, Proc. Sympos. Pure Math., vol. 9, Amer. Math. Soc., Providence, R.I., 1965, pp. 99-103. MR 34 \#4373.

10. E. L. Spitznagel, Jr., Terminality of the maximal unipotent subgroups of Chevalley groups, Math. Z. 103 (1968), 112-116. MR 37 \#2867.

11. - Structure and terminality of the maximal unipotent subgroups of Steinberg groups, Illinois J. Math. 13 (1969), 400-405. MR 39 \#4284.

12. M. Stein, Chevalley groups over commutative rings, Bull. Amer. Math. Soc. 77 (1971), 247-252.

13. R. Steinberg, Variations on a theme of Chevalley, Pacific J. Math. 9 (1959), 875891. MR 22 \#79.

14. - Lectures on Chevalley groups, Yale University, New Haven, Conn., $1967 / 68$.

Department of Mathematics, University of California, Riverside, California 92502

Department of Mathematics, University of Connecticut, Storrs, Connecticut 06268 